The Institution of

Engineering and Technology

\title{
RPG 2022
}

\section{Renewable Power Generation}

22-23

September 2022

Savoy Place,

London
Meeting net

zero carbon

Call for papers deadline:

10 December 2021

$f$ in $y$ \#RPGLondon 


\title{
One-day ahead wind speed/power prediction based on polynomial autoregressive model
}

ISSN 1752-1416

Received on 12th December 2016

Revised 14th April 2017

Accepted on 12th June 2017

E-First on 27th July 2017

doi: 10.1049/iet-rpg.2016.0972

www.ietdl.org

\author{
Oktay Karakuş ${ }^{1}$, Ercan E. Kuruoğlu², Mustafa A. Altınkaya ${ }^{1}$ \\ ${ }^{1}$ Department of Electrical-Electronics Engineering, Izmir Institute of Technology, Izmir, Turkey \\ ${ }^{2}$ Institute of Science and Technology of Information 'A. Faedo', CNR, G. Moruzzi 1, 56124 Pisa, Italy \\ 凶E-mail: oktaykarakus@iyte.edu.tr
}

\begin{abstract}
Wind has been one of the popular renewable energy generation methods in the last decades. Foreknowledge of power to be generated from wind is crucial especially for planning and storing the power. It is evident in various experimental data that wind speed time series has non-linear characteristics. It has been reported in the literature that nonlinear prediction methods such as artificial neural network (ANN) and adaptive neuro fuzzy inference system (ANFIS) perform better than linear autoregressive (AR) and AR moving average models. Polynomial AR (PAR) models, despite being non-linear, are simpler to implement when compared with other non-linear AR models due to their linear-in-the-parameters property. In this study, a PAR model is used for one-day ahead wind speed prediction by using the past hourly average wind speed measurements of Çeşme and Bandon and performance comparison studies between PAR and ANN-ANFIS models are performed. In addition, wind power data which was published for Global Energy Forecasting Competition 2012 has been used to make power predictions. Despite having lower number of model parameters, PAR models outperform all other models for both of the locations in speed predictions as well as in power predictions when the prediction horizon is longer than $12 \mathrm{~h}$.
\end{abstract}

\section{Introduction}

Wind speed or wind power prediction plays a key role in wind power management for governments and energy companies. A reliable and highly precise prediction provides better control and planning of power, e.g. daily and hourly scheduling, transmission, storage and balance of power and other managing related operations [1]. When it is not possible to measure and apply prediction operation directly to wind power, wind speed prediction becomes important, since it is directly related to power. Short-term wind speed prediction is crucial for preventing damage to wind farms due to sudden wind gust, whereas long-term prediction serves especially for planning of generated power. Moreover, predicting directly from power time series eliminates extra errors resulting from cubic conversion of erroneous wind speed estimates.

Several forecasting horizons can be used in power markets for both management and trading issues. For example, for management issues, from 2 to 3 days ahead predictions are required for systems such as steam turbines etc. Up to $6 \mathrm{~h}$ predictions can be enough for systems with lower complexity such as gas turbines. Predictions which lie within $48 \mathrm{~h}$ are generally required for trading issues. However, longer or shorter horizons can be chosen according to the application and area in which the predictions will be used [2-4].

Wind speed/power forecasting models can be listed in two groups which are physical and statistical models. Physical models perform wind forecasting by using meteorological and atmospherical instances. Numerical weather prediction is a popular approach in physical modelling which uses meteorological instances and performs predictions via solving complex mathematical models about physical equations of the atmosphere. Its performance is relatively well especially in stable weather conditions and is lower especially for short-term, because of the uncertainties on initial atmospheric conditions and parameterisations $[5,6]$.

There are several statistical models for wind speed/power prediction in the literature which can be listed as the persistence model [7], linear time series-based models [8-10], artificial neural networks (ANNs) $[11,12]$ and adaptive neuro fuzzy inference systems (ANFISs) [13, 14]. Persistence model is the simplest one among these models and can be defined as the naive predictor in meteorological studies [7] which makes predictions under the assumption that the future value (prediction) will be the same as the current measurement.

Linear time series-based models have been frequently used in previous works on wind speed/power prediction. Those works which use autoregressive (AR), AR moving average (ARMA) and $A R$ integrated MA (ARIMA) or seasonal-ARIMA-based models have demonstrated a limited degree of success in predicting shortterm (especially from $10 \mathrm{~min}$ up to $6 \mathrm{~h}$ ) wind speed/power. In particular, $\operatorname{ARIMA}(2,0,0)$ [equivalently $\operatorname{AR}(2)$ ] model has performed better than feed forward ANN with different validation techniques at prediction horizon between $10 \mathrm{~min}$ and $4 \mathrm{~h}$ [8]. Furthermore, ARMA models have been compared with ANN models in short-term prediction [9] and achieved lower values of error measures than the ANN models in the simulations. In another study [10], short-term prediction performance of ARMA models has been compared with that of the persistence model and these models performed better than persistence at different locations in Spain. These studies have clearly showed that linear time series models achieve considerably good performance and they are preferable models for short-term over persistence model and several ANN.

When the prediction horizon is longer than $6 \mathrm{~h}$, linear time series methods fall short due to the non-linear character of wind speed/power. To overcome this, various non-linear methods such as ANN and ANFIS are used for both short and long-term prediction in the literature. These methods outperform linear time series methods for various wind speed and wind power data from various locations in the world. Data mining approaches and hybrid systems have been proposed in studies [15-17] in order to predict wind speed/power up to 1-3 days and they outperform linear and some specific non-linear methods. Furthermore, Gaussian processes [18], random forest and bagging trees $[19,20]$ are also utilised in prediction studies.

Non-linear time series methods are also used for wind speed/ power prediction studies. A Volterra model-based prediction scheme trained by a recursive ANN (R-ANN) has been utilised in [21] and a pattern-based hybrid wind speed forecasting scheme with a Hammerstein-AR model has been proposed [1]. In addition, 
in other studies nonlinear autoregressive moving average with exogenous inputs (NARMAX) models have been proposed [22, 23].

Polynomial AR (PAR) models are based on the Volterra series expansion (1), which has been applied successfully in areas such as biological systems [24], industrial plant control systems [25], communications [26] and seismology [27]. PAR processes differ from the other non-linear AR processes in that they are linear-inthe-parameters and thus many mathematical applications developed for linear models can be employed without much difficulty [28].

In a preliminary work presented as conference communication [29], PAR models performance was compared with linear models. In this paper, we extend our comparisons with state-of-the-art nonlinear models such as ANN and ANFIS models whose number of parameters and computational complexities are relatively higher than the proposed model, PAR. We also enrich our simulations on various experimental data: in addition to hourly average wind speed data of Çeşme (İzmir, Turkey), data from another location in USA, Bandon (Oregon) are used for this paper. Performance comparison is provided visually for two different periods (JulyAugust-September and January-February-March) using error measures such as normalised root mean square error (NRMSE), normalised mean absolute percentage error (NMAPE) and bias.

In this paper, in addition to wind speed predictions, we have performed a wind power prediction study directly using wind power time series. For this purpose, globally reachable wind power measurements which were published for Global Energy Forecasting Competition 2012 [30] have been utilised for the prediction study and performances of models have been compared by error metrics NRMSE, NMAPE and bias.

Rest of this paper is organised as follows: PAR models and representative state-of-the-art models, ANN and ANFIS models are examined in Section 2. Section 3 exhibits estimation methods and error measures used in prediction procedure. Wind data information, the simulation results and error analysis have been provided in Section 4. Section 5 concludes this paper with a discussion of experimental results.

\section{Models}

\subsection{PAR models}

PAR models can be represented as [28]

$$
\begin{aligned}
x(l)= & \mu+\sum_{i}^{k} a_{i}^{(1)} x(l-i)+\sum_{i}^{k} \sum_{j}^{k} a_{i, j}^{(2)} x(l-i) x(l-j) \\
& +\cdots+\sum_{i, \ldots}^{k, \ldots} a_{i, \ldots}^{(p)} x(l-i) \ldots+\epsilon(l)
\end{aligned}
$$

where $\mu$ is the intercept, $\epsilon(l)$ is an independent and identically distributed (i.i.d.) excitation sequence with distribution $\mathscr{N}\left(0, \sigma_{\epsilon}^{2}\right)$, $a_{i}^{(1)}, a_{i, j}^{(2)}$ and $a_{i, \ldots}^{(p)}$ are coefficients for first, second and $p$ th-order polynomials, respectively, $p$ is the non-linearity degree and $k$ is the AR order. We represent a PAR model with the notation: $\mathrm{P}^{(p)} \mathrm{AR}(k)$.

A $\mathrm{P}^{(p)} \mathrm{AR}(k)$ model given by (1) can be expressed in matrixvector form by using the linear-in-the-parameters property

$$
\boldsymbol{x}=\mu+\boldsymbol{X} \boldsymbol{a}^{(p, k)}+\boldsymbol{\epsilon}
$$

where $\boldsymbol{x}$ is an $n \times 1$ data vector, $\boldsymbol{X}$ is an $n \times w$ matrix of past samples and polynomial products of the data, $\boldsymbol{a}^{(p, k)}$ is a $w \times 1$ coefficient vector, $\boldsymbol{\epsilon}$ is an $n \times 1$ vector of excitation sequence, $n$ is the data length and $w$ is the number of model coefficients. The coefficient vector $\boldsymbol{a}^{(p, k)}$ and the matrix $\boldsymbol{X}$ can be defined as in (3) and (4), respectively

$$
\boldsymbol{a}^{(p, k)}=[a_{1}^{(1)}, a_{2}^{(1)}, \ldots, a_{k}^{(1)}, a_{1,1}^{(2)}, a_{1,2}^{(2)}, \ldots, a_{k, k}^{(2)}, \ldots, \underbrace{a_{k, k, \ldots, k}^{(p)}}_{p \text { th order }}]^{\mathrm{T}}
$$

(see (4)) AR models are subsets of PAR models. So, $\mathrm{P}^{(p)} \mathrm{AR}(k)$ models are equivalent to $\operatorname{AR}(k)$ models when the non-linearity degree, $p$, is set to 1 .

\subsection{ANN models}

ANNs are statistical learning models which are inspired by central nervous system of animals and composed of elements called nodes, also known as neurons. Interconnected neurons exchange messages between each other. These systems are trained via various training algorithms and this learning process updates weights of interconnections. ANN are formed of layers which are composed of a number of interconnected neurons and every neuron contains an activation function which is a zero memory non-linear function. Inputs are fed to the input layer which is connected to one or more hidden layers. The actual processing is provided by hidden layers via a system of weighted connections. The output layer which is linked to the hidden layers provides the final result. Five different ANN structures will be used in this paper. These are: multilayer FF (MLFF), multilayer perceptron (MLP), Elman recurrent, radial basis function (RBF) and linear (for details, see studies [13, 31]). Inputs are past hourly average wind speed/power data and the output is the predicted wind speed/power data. For example, if the number of inputs is $r$, then the corresponding inputs for the onestep ahead predicted output $\hat{y}(l)$ are $\{y(l-1), y(l-2), \ldots, y(l-r)\}$.

\subsection{ANFIS models}

ANFIS models are combinations of two statistical systems and create a successful hybrid system of neural networks and fuzzy inference systems. The working principle of an ANFIS model consists of two steps [13]: (i) the system is trained in a similar manner with ANN and (ii) trained system will then operate as a fuzzy inference system. ANFIS model is based on Takagi-Sugeno fuzzy inference system [32]. Since ANFIS integrate both ANN and fuzzy logic principles, the advantages of both systems are integrated in a single system. Fuzzy interference system (FIS) part has the capability to approximate non-linear functions via a set of if-then rules [33]. Basically, ANFIS are constructed with five layers. Each layer is formed of nodes which are described by the node functions. The outputs of previous layers are inputs of next layers. ANFIS use either backpropagation or a combination of least-squares (LSs) estimation and backpropagation for the membership function (MF) parameter estimation. In addition, ANFIS models have both linear parameters of the consequent MF and non-linear parameters of the antecedent MF. The number of consequent parameters depends on the number of rules, which in turn depends on the number of inputs. However, the number of antecedent parameters depends on the number of MFs per input and the MF type in addition to the number of inputs [34].

\section{Estimation methods}

\subsection{Multistep ahead prediction}

Prediction study in this paper requires a multistep ahead prediction procedure in order to make predictions up to $24 \mathrm{~h}$ ahead.

$$
\boldsymbol{X}=\left[\begin{array}{cccccccccc}
x(0) & x(-1) & \ldots & x(1-k) & x^{2}(0) & x(0) x(-1) & \ldots & x^{2}(1-k) & \ldots & x^{p}(1-k) \\
x(1) & x(0) & \ldots & x(2-k) & x^{2}(1) & x(1) x(0) & \ldots & x^{2}(2-k) & \ldots & x^{p}(2-k) \\
\vdots & \vdots & \ddots & \vdots & \vdots & \vdots & \ddots & \vdots & \ddots & \vdots \\
x(n-1) & x(n-2) & \ldots & x(n-k) & x^{2}(n-1) & x(n-1) x(n-2) & \ldots & x^{2}(n-k) & \ldots & x^{p}(n-k)
\end{array}\right]
$$




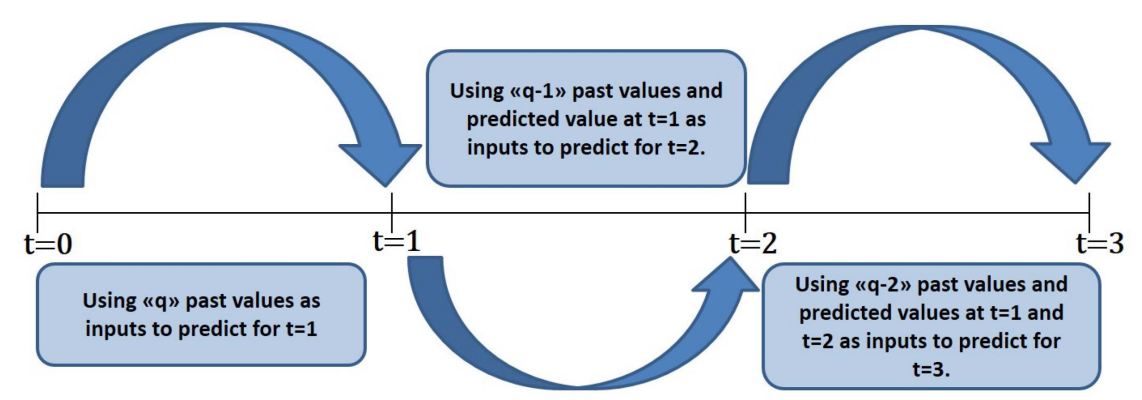

Fig. 1 Three-step ahead prediction example

Predictions have been made for all the models in this paper (AR, PAR, ANN and ANFIS) by employing the procedure explained below.

A time series $\boldsymbol{Y}=\left[y_{1}, y_{2}, \ldots, y_{n}\right]$ can be defined as a sequence of recorded observations at each time step. If we take a segment of data with length $q$ up to time $l$, this segment will be $\boldsymbol{Y}_{l-q+1}^{l}=\left[y_{l}, y_{l-1}, \ldots, y_{l-q+1}\right] . \quad T$-step ahead prediction of $\boldsymbol{Y}$ is represented as $\boldsymbol{Y}_{l+1}^{l+t}$ and will be a function of $q$ past values. This can be defined as [35]

$$
\boldsymbol{Y}_{l+1}^{l+t}=f\left(y_{l}, y_{l-1}, \ldots, y_{l-q+1}\right)=f\left(\boldsymbol{Y}_{l-q+1}^{l}\right)
$$

The keypoint in multistep ahead prediction is to use only the measurements up to time $l$. If measurements at time $l+1, l+2, \ldots$ are needed, predicted values will be used instead of these measurements. An example of three-step ahead prediction have been shown in Fig. 1.

\subsection{Coefficient estimation of PAR models}

The non-linear $L S$ (NLS) estimation generates optimal PAR coefficient estimates under the assumption that the excitation, $\epsilon(l)$, are i.i.d. Gaussian random variables. PAR model coefficients, $\boldsymbol{a}^{(p, k)}$ which are defined in (3) have been estimated by using the training data for all data sets by employing the NLS method, since the data matrix $\boldsymbol{X}$ includes polynomial products of past data samples. Under the assumption that the model orders $p$ and $k$ are known initially, NLS estimates of PAR model coefficients by [36]

$$
\hat{\boldsymbol{a}}_{\mathrm{NLS}}^{(p, k)}=\left(\boldsymbol{X}^{\mathrm{T}} \boldsymbol{X}\right)^{-1} \boldsymbol{X}^{\mathrm{T}} \boldsymbol{x}
$$

On the other hand, when $\epsilon(l)$ have heavier tails than a Gaussian distribution, computing the variance may not be possible and other approaches can be applied instead of NLS. Using minimum dispersion-based estimation methods instead of minimum MSE appears as good alternatives here. Non-linear polynomial least $L-p$ norm-based estimation methods such as non-linear iteratively reweighted LS and normalised polynomial least mean Pth power, perform optimal coefficient estimations for PAR processes with $\alpha$ stable or generalised Gaussian innovations [28].

In this paper, we assume a Gaussian excitation for PAR models for simplicity and apply NLS directly since PAR models are linearin-the-parameters models.

\subsection{Performance metrics}

There are various error metrics which have been used in the literature to compare prediction performances of the methods. In this paper, we use NRMSE, NMAPE and bias metrics in order to measure the performances to visualise both $L_{1}$-norm (NMAPE) and $L_{2}$-norm (NRMSE)-based errors and the bias. NRMSE provides a good measure of distortion when the error is Gaussian distributed while NMAPE is successful in dealing with data containing outliers. We have used bias in order to see whether the predictions are under or overestimated. These metrics are defined as

$$
\begin{aligned}
\text { NRMSE } & =\sqrt{\frac{1}{n} \sum_{i=1}^{n}\left(\frac{x_{i}-\hat{x}_{i}}{\max (\boldsymbol{x})}\right)^{2}} \\
\mathrm{NMAPE} & =\frac{1}{n} \sum_{i=1}^{n} \frac{\left|x_{i}-\hat{x}_{i}\right|}{\max (\boldsymbol{x})} \times 100 \\
\text { bias } & =\frac{1}{n} \sum_{i=1}^{n}\left(x_{i}-\hat{x}_{i}\right)
\end{aligned}
$$

where $\boldsymbol{x}$ and $\hat{\boldsymbol{x}}$ are the test and the predicted time series of length $n$, respectively. Best results are shown with lower values of NRMSE and NMAPE.

\section{Experimental analysis}

\subsection{Data}

In this paper, wind speed data which are measured at Çeşme (İzmir, Turkey) and Bandon (Oregon, USA) are used to make predictions up to $24 \mathrm{~h}$ ahead. Generally, accepted procedure in the literature is to use hourly average values in the studies. In addition, our data sets include only hourly averages. The term 'hourly average' corresponds to the average of the samples obtained within an hour with a sampling period of several minutes. Using hourly average values provides computationally cheaper operations in the same time interval. Moreover, short averages generate erratic values than the longer. Thus, using hourly averages is appropriate and the smoothing effect generated by this averaging is assumed to be negligible [37].

In particular, first data set is hourly average wind speed data measured in Çeşme at an altitude of $10 \mathrm{~m}$ by Turkish State Meteorological Service. Bandon data are also hourly average wind speed data and measured by AgriMet (Cooperative Agricultural Weather Network) [38]. The Bandon site is located at $25 \mathrm{~m}(80 \mathrm{ft})$ elevation and at the West coast of Oregon, USA.

For wind speed prediction studies, two 3 months long periods have been selected as test periods in order to measure the performance of the models in different times of the year. In particular, test period 1 covers January-February-March and period 2 covers July-August-September and model performances have been measured both for the first months and whole 3 months of the periods. Measurements from 12 and 24 months before these test periods are selected as candidate training data sets. Then, we perform a comparison of the informative content for training sets with different lengths (i.e. 1 and 2 years) using entropy to quantify the information content of the sequences. As a result, the entropy values for both of the training sets are very close to each other for all the data sets. Hence, using shorter training period will be more appropriate in the sense of computational complexity.

Fig. 2 shows hourly average wind speed distributions of test months for two locations for both training and test data sets. Also, both of the subfigures depict that the distributions for training and test data have similar characteristics. Specifically, Bandon test data for period 2 have quite lower values than training; however, this fact has been compensated by the training data.

Wind power measurements used in this paper are from a globally reachable data set which was published for Global Energy Forecasting Competition 2012 [30] including wind power 


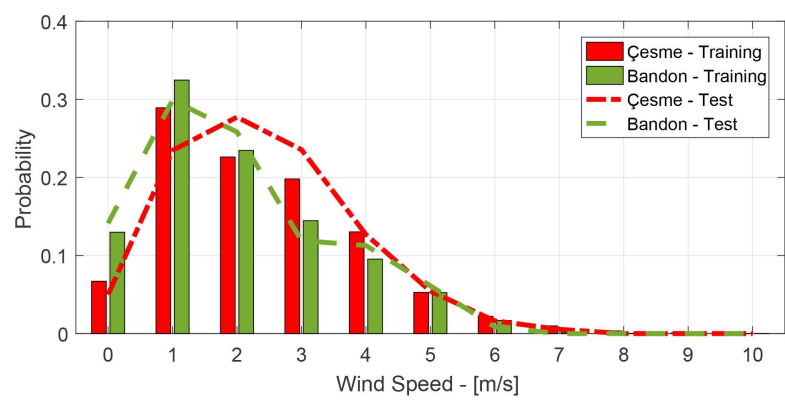

Fig. 2 Wind speed histograms

(a) Period 1, (b) Period 2

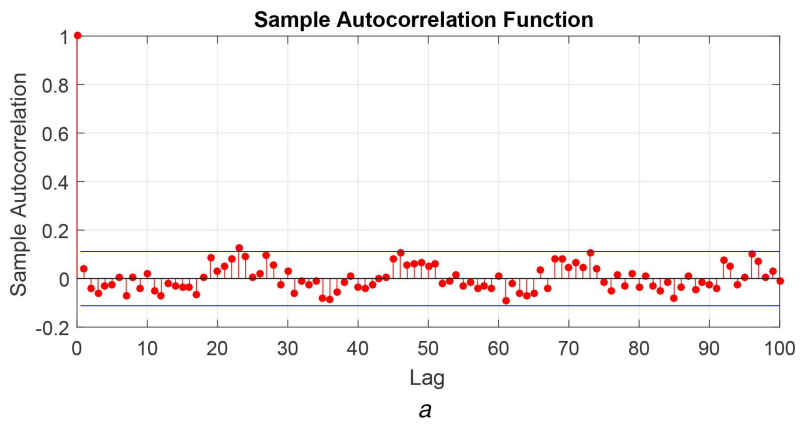

Fig. 3 Residual autocorrelations for randomly selected periods with length of 720 samples. Limits in these figures refer to $\pm 3 \sigma$ (a) Çeşme (period 1), (b) Power (period 2)

measurements from several wind farms and electricity load data. Wind power measurements of a single wind farm ( $w p 1$ in the data set excel sheet) have been selected and used in the wind power predictions. Testing data periods are the same dates with wind speed data sets and historical data 12 months before these periods have been selected as training data.

\subsection{Modelling results}

Each model explained in previous sections is applied in a training procedure with 1 year length wind speed/power data and 1 month length wind speed/power test data. Both training and test data are normalised in a range between 0 and 1 in order to apply prediction procedures at the same interval for every data set.

4.2.1 Simulated PAR models: To decide the best AR and PAR models in this paper, Akaike information criterion (AIC) and Bayesian information criterion (BIC) methods have been performed to select PAR or AR models for wind speed/power predictions where model space includes nine different PAR models (for $p=1,2,3$ and $k=1,2,3$ ). However, estimated orders by AIC or BIC did not provide the best results in terms of prediction error. Therefore, the 'best' PAR and AR models were selected by performing an exhaustive search on models comparing their $24 \mathrm{~h}$ ahead prediction performances (Please see [39] to estimate best PAR model via AIC and BIC.).

For Çeşme wind speed, $\mathrm{P}^{(2)} \mathrm{AR}(3)$ and $\mathrm{AR}(3)$ models have been found to be the best PAR and AR models according to their NRMSE values for both $24 \mathrm{~h}$ ahead prediction, where for Bandon, $\mathrm{P}^{(2)} \mathrm{AR}(2)$ and $\mathrm{AR}(2)$ models have been selected. For power prediction studies, $\mathrm{P}^{(2)} \mathrm{AR}(1)$ and $\mathrm{AR}(1)$ models achieve the lowest NRMSE values.

PAR/AR model coefficients for each model in question have been estimated via NLS method by using the exhaustively estimated model orders. Moreover, in training period, PAR models have been tested for zero and non-zero intercepts and PAR models with zero intercept achieve better prediction results than that of non-zero intercept. That is why the intercept, $\mu$, has been selected as zero for all the data sets in this paper. PAR model excitation sequence $\epsilon_{n}$ is a Gaussian process with mean 0 and variance $\sigma_{\epsilon}^{2}$.
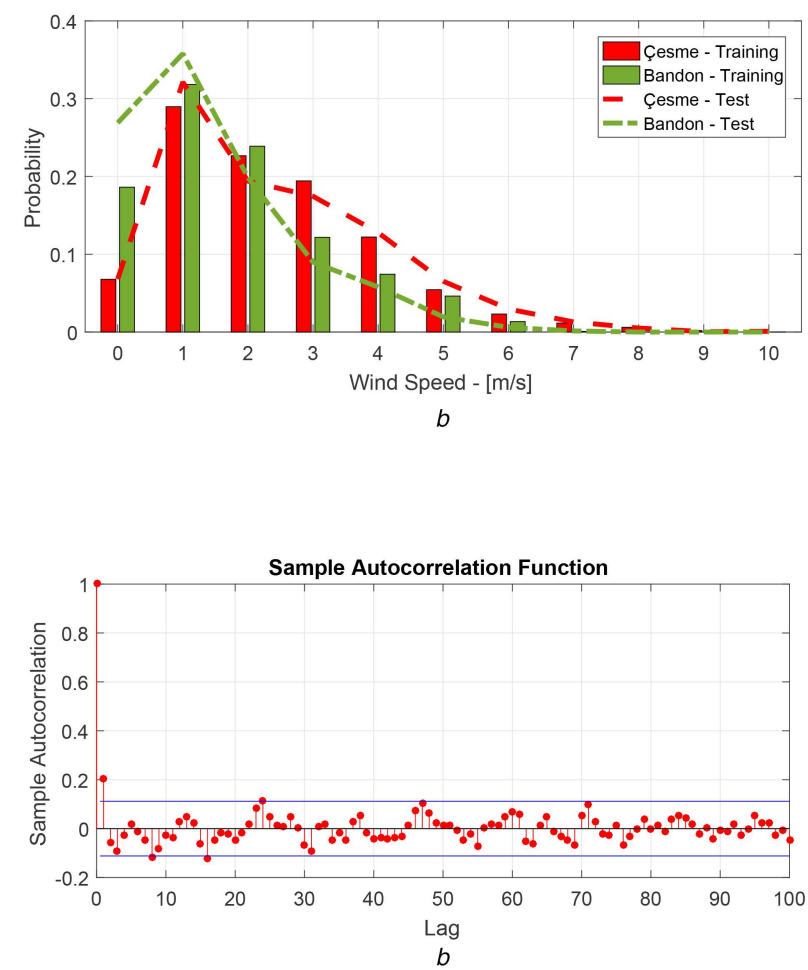

Excitation variance values for each data set have been estimated in training process.

By using all these estimated and initially defined parameters, PAR model equation in (1) has been used to make predictions by taking $k$ past wind speed samples as its input. About 1000 Monte Carlo simulations have been performed and averages have been used as the predictions in order to obtain stable results.

\subsection{Validation of PAR models}

To validate the PAR models estimated for prediction, first a statistical significance study has been run based on the residual autocorrelations. Each training data set has a length of 8760 samples and to perform residual autocorrelations a 1 month long (720 samples) period inside of the training set has been randomly selected for each data set.

In Fig. 3, residual autocorrelations have been depicted for two example data sets (Çeşme Period 1 and Power Period 2). Examining both of the subfigures, it can be clearly stated that the residual autocorrelations are generally below the limits and are contained. However, they both show a cyclic character with a period of $24 \mathrm{~h}$ (1 day). Notwithstanding, correlation values of the residuals for each lag are low. Not to repeat the same results, we only show 2 out of 6 figures. All other four data sets show same characteristics such as the examples. Thus, we can extend the comments above to all data sets that we utilised in this paper.

Apart from residual autocorrelations, PAR models obtain reasonable fitting results which are measured using adjusted- $R^{2}$ and $F$-test. Both tests measure the fitting performance of an estimated model. Estimated PAR models $\left[\mathrm{P}^{(2)} \mathrm{AR}(3)\right.$ for Çeşme, $\mathrm{P}^{(2)} \mathrm{AR}(2)$ for Bandon and $\mathrm{P}^{(2)} \mathrm{AR}(1)$ for Power] for each data set have achieved at least $65 \%$ adjusted- $R^{2}$ score while rival methods also achieve nearly the same values. Although all the models have $p$-values lower than 0.0001 after $F$-test, $F$-score of PAR model is higher and it can be stated that its fitting performance is slightly better than the others.

4.3.1 Simulated ANN and ANFIS models: ANN models are implemented by using the parameters given in Table 1 . ANN and ANFIS model structures are created in MATLAB ${ }^{\mathbb{R}}$, $s$ ANN and FIS toolboxes and models are oriented to minimise the MSE. Some of 
the models and their parameters for ANN and ANFIS models have been taken from a previous study [13] on wind prediction using several ANN and ANFIS models. In addition to the ANN models in Table 1, two ANFIS structures with three inputs have been implemented, where both use linear output MF and hybrid optimisation method. The only difference is the MF types in question, where ANFIS-1 uses generalised bell and ANFIS-2 employs triangular-shaped MFs.

Number of inputs have been decided on after an exhaustive search for each model (ANN and ANFIS) between 1 and 6 by choosing the one giving the best performance. Consequently, number of inputs have been decided as three in wind speed prediction for both ANN and ANFIS for both locations. For wind power prediction, number of inputs are selected for ANN models as one for both of the test months.

Table 2 depicts the number of adjustable parameters for each simulated model. Linear models AR and linear-ANN (L-ANN) have a small number of parameters. PAR models are non-linear models but their complexity is low when compared with other nonlinear models in terms of number of adjustable parameters. In addition, complexity of the NLS used for estimating the parameters of PAR and AR models is lower than those of the training algorithms which ANN and ANFIS models have employed.

\subsection{Wind speed prediction results}

Between Tables 3 and 6 show the performance of all the implemented models for four different cases which are Çeşmeperiod1, Bandon-period1, Çeşme-period 2 and Bandon-period2. In these tables, one PAR model is indicated for each case. These models are the best PAR models which achieve the lowest NRMSE value for $24 \mathrm{~h}$ ahead prediction as stated in Section 4.2.1. Values for the best models for each error metric have been depicted as bold in the tables.

Examining the values in Table 3, the performance of $\mathrm{P}^{(2)} \mathrm{AR}(3)$ model is clearly much better than all other models when prediction horizon is longer than $6 \mathrm{~h}$. RBF, MLP and ANFIS-2 have achieved the second best performance after PAR models and they are preferable to all other models for a longer prediction horizon. For all prediction horizons, all the models, except $\mathrm{P}^{(2)} \mathrm{AR}(3)$ have underestimated the wind speed (bias values are over zero). Below $12 \mathrm{~h}$ linear model AR(3) model is preferable which is a fact that

$\underline{\text { Table } 1 \text { ANN simulation parameters }}$

\begin{tabular}{|c|c|c|c|c|c|}
\hline & MLFF & $\mathrm{RBF}^{\mathrm{a}}$ & MLP & Elman $^{b}$ & L-ANN \\
\hline training function & TRAINLMe & - & TRAINLM & TRAINLM & TRAINGDX \\
\hline learning function & LEARNGD $^{\mathrm{e}}$ & LEARNGD & LEARNPe & LEARNGD & LEARNGD \\
\hline number of layers & 4 & 3 & 3 & 3 & 2 \\
\hline neurons - inputs $^{c}(I)$ & $3 / 1$ & $3 / 1$ & $3 / 1$ & $3 / 1$ & $3 / 1$ \\
\hline neurons - hidden $1^{\mathrm{d}}\left(H_{1}\right)$ & $10 / 20$ & variable (maximum 30 ) & $10 / 20$ & $10 / 20$ & - \\
\hline neurons - hidden $2^{\mathrm{d}}\left(\mathrm{H}_{2}\right)$ & $10 / 20$ & - & - & - & - \\
\hline notation & $I-H_{1}-H_{2}-1$ & $I-H_{1}-1$ & $I-H_{1}-1$ & $I-H_{1}-1$ & $1-1$ \\
\hline activation function (hidden) & TANSIG $^{\mathrm{e}}$ & RADBAS $^{\mathrm{e}}$ & TANSIG & TANSIG & - \\
\hline activation function (output) & PURELIN & PURELIN & PURELIN & PURELIN & - \\
\hline epochs & 200 & 200 & 200 & 200 & 200 \\
\hline
\end{tabular}

${ }^{\mathrm{a}}$ Neurons are iteratively added to the RBF network one by one until the sum-squared error falls beneath 0.005 or a maximum number of neurons have been reached.

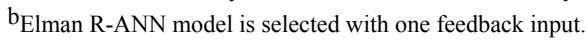

${ }^{c}$ One input neuron has been used for wind power predictions.

$\mathrm{d}_{\text {About }} 20$ hidden neurons have been used for wind power predictions.

e LM: Levenberg-Marquardt algorithm, GDX: gradient descent with momentum and adaptive learning rate backpropagation, GD: gradient descent, $P$ : perceptron, TANSIG:

hyperbolic tangent sigmoid and RADBAS: RBF.

Table 2 Number of adjustable parameters for the simulated models

\begin{tabular}{lccccccc}
\hline Model & $\begin{array}{c}\text { Number of } \\
\text { parameters }\end{array}$ & Model & $\begin{array}{c}\text { Number of } \\
\text { parameters }\end{array}$ & Model & $\begin{array}{c}\text { Number of } \\
\text { parameters }\end{array}$ & $\begin{array}{c}\text { Model } \\
\text { Number of } \\
\text { parameters }\end{array}$ \\
\hline $\operatorname{AR}(1)$ & 1 & $P^{(2)}$ AR(3) & 9 & L-ANN (1-1) & 2 & MLFF (3-10-10-1) & 161 \\
$\operatorname{AR}(2)$ & 2 & Elman (3-10-1) & 151 & L-ANN (3-1) & 4 & MLFF (1-20-20-1) & 481 \\
$\operatorname{AR}(3)$ & 3 & Elman (1-20-1) & 461 & MLP (3-10-1) & 51 & ANFIS-1 & 135 \\
$\mathrm{P}^{(2)} \mathrm{AR}(1)$ & 2 & RBF (1-Var-1) & $31-91$ & MLP (1-20-1) & 61 & ANFIS-2 & 135 \\
$\mathrm{P}^{(2)} \mathrm{AR}(2)$ & 5 & RBF (3-Var-1) & $51-151$ & & & \\
\hline
\end{tabular}

Table 3 Performance comparison of Çeşme wind speed time series for the test period 1

\begin{tabular}{|c|c|c|c|c|c|c|c|c|c|c|c|c|}
\hline & \multicolumn{9}{|c|}{1 month test } & \multicolumn{3}{|c|}{3 months test } \\
\hline & \multicolumn{3}{|c|}{$6 \mathrm{~h}$} & \multicolumn{3}{|c|}{$12 \mathrm{~h}$} & \multicolumn{3}{|c|}{$24 \mathrm{~h}$} & \multicolumn{3}{|c|}{$24 h$} \\
\hline & NRMSE & NMAPE & Bias & NRMSE & NMAPE & Bias & NRMSE & NMAPE & Bias & NRMSE & NMAPE & Bias \\
\hline $\mathrm{P}^{(2)} \mathrm{AR}(3)$ & 0,0554 & 5,6688 & 0,0001 & 0,0762 & 8,2396 & $-0,0056$ & 0,1026 & 11,7231 & $-0,0187$ & 0,1015 & 11,7429 & $-0,0188$ \\
\hline $\operatorname{AR}(3)$ & 0,0511 & 5,5591 & 0,0361 & 0,0801 & 9,2375 & 0,0649 & 1297 & 15,5834 & 0,1108 & 0,1294 & 15,4769 & 0,1100 \\
\hline ILFF & 0,0638 & 6,6579 & 0,0084 & 0,0882 & 9,4067 & 0,0137 & 48 & 12,4311 & 0,0183 & 0,1155 & 12,8892 & 0,0165 \\
\hline ILP & 0,0653 & 6,8056 & 0,0047 & 0,0894 & 9,4993 & 0,0072 & 0,1145 & 12,3899 & 0,0083 & 0,1152 & 12,8364 & 0,0064 \\
\hline Elman & 0,0666 & 7,0689 & 0,0034 & 0,0920 & 9,8633 & 0,0047 & 0,1160 & 12,6288 & 0,0046 & 0,1169 & 13,0848 & 0,0026 \\
\hline -ANN & 0,0800 & 8,7943 & 0,0035 & 0,1045 & 11,4765 & 0,0052 & 0,1245 & 13,6713 & 0,0067 & 0,1257 & 14,1844 & 0,0046 \\
\hline RBF & 0,0662 & 6,7510 & 0,0119 & 0,0908 & 9,3135 & 0,0203 & 0,1147 & 12,0531 & 0,0285 & 0,1151 & 12,5064 & 0,0264 \\
\hline ANFIS & 0,0677 & 6,8119 & 0,0156 & 0,0926 & 9,4747 & 0,0279 & 0,1178 & 12,1115 & 0,0398 & 0,1177 & 12,5584 & 0,0378 \\
\hline ANFIS-2 & 0,0612 & 6,5445 & 0,0086 & 0,0863 & 9,4519 & 0,0158 & 0,1148 & 12,5259 & 0,0234 & 0,1139 & 12,9248 & 0,0209 \\
\hline
\end{tabular}


supports the previous studies [8-10] about wind speed prediction that linear models are more appropriate models for short-term prediction, whereas non-linear models work better for long-term wind speed prediction.

Prediction performance for Bandon in test period 1 as shown in Table 4 provides nearly the same results as that for Çeşme. $\mathrm{P}^{(2)}$ $\mathrm{AR}(2)$ model appears to be preferable to all other models. MLFF is the best performing ANN model and ANFIS-1 is the best performing ANFIS model for Bandon data at $24 \mathrm{~h}$ ahead prediction. For all prediction horizons, all the models' predictions have been underestimated for Bandon.

In Table 5, it has been shown that the performance of $\mathrm{P}^{(2)} \mathrm{AR}(3)$ outperforms all other models when prediction horizon is over $12 \mathrm{~h}$. RBF and linear AR(3) perform well for all horizons in test period 2. Generally, most of the models have overestimated the wind speed (bias values are above zero). Moreover, in Table 6, the proposed model $\mathrm{P}^{(2)} \mathrm{AR}(2)$ shows remarkable performance in all the cases. In addition, linear AR(2) model performs as the second best model when all other models achieve very low prediction performance in test period 2 in Bandon site. Bias results appear similar with Çeşme test period 2 results. Specifically, most of the models overestimated wind speed in all the prediction horizons.

Figs. $4 a$ and $b$ show the $24 \mathrm{~h}$ ahead prediction results versus observed test data in a randomly selected week in July. Simulated models are divided into four groups, namely PAR, ANN (MLFF, MLP, Elman and RBF), ANFIS and linear (AR and L-ANN). The best model for prediction horizon of $24 \mathrm{~h}$ from each group is selected and plotted. PAR models appear remarkable for this prediction horizon when ANFIS and ANN models did not notably capture their performance. Figs. $4 c$ and $d$ show percentage of NRMSE improvement obtained by the PAR model with respect to other models. Observing the values in Fig. $4 c$ for all the prediction horizon values over $12 \mathrm{~h}$, PAR model improves the prediction performance in NRMSE over ANN and ANFIS models by about $20 \%$. In Fig. $4 d$, again PAR model has an improvement over ANN and ANFIS models by about $35 \%$.

In Figs. $5 a$ and $b$, the $24 \mathrm{~h}$ ahead prediction results for January have been shown for both locations. PAR model outperforms all other models again for winter. Figs. $5 c$ and $d$ show NMAPE improvement obtained by the PAR model with respect to other models. NMAPE improvement means the reduction in NMAPE obtained by PAR with respect to those of the other simulated

Table 4 Performance comparison of Bandon wind speed time series for the test period 1

\begin{tabular}{|c|c|c|c|c|c|c|c|c|c|c|c|c|}
\hline & \multicolumn{9}{|c|}{1 month test } & \multicolumn{3}{|c|}{3 months test } \\
\hline & \multicolumn{3}{|c|}{$6 \mathrm{~h}$} & \multicolumn{3}{|c|}{$12 \mathrm{~h}$} & \multicolumn{3}{|c|}{$24 \mathrm{~h}$} & \multicolumn{3}{|c|}{$24 h$} \\
\hline & NRMSE & NMAPE & Bias & NRMSE & NMAPE & Bias & NRMSE & NMAPE & Bias & NRMSE & NMAPE & Bias \\
\hline $\mathrm{P}^{(2)} \mathrm{AR}(2)$ & 0,0683 & 6,1242 & 0,0235 & 0984 & 9,4282 & 0,0293 & 0,1291 & 13,4536 & 0272 & 0,1122 & 1,8619 & 0,0026 \\
\hline $\mathrm{AR}(2)$ & 0,0594 & 7273 & 0,0498 & 1069 & 12,2032 & 0,0907 & 798 & 20,5896 &, 1532 & 0,1574 & 17,1953 & 0,1278 \\
\hline MLFF & 0,0864 & 8,8088 & 0,0311 & 0,1332 & 13,4365 & 0,0489 & 0,1660 & 16,9221 & 0,0566 & 0,1505 & 15,7561 & 0,0153 \\
\hline MLP & 0,0865 & 9,1269 & 0,0349 & 0,1377 & 14,3080 & 0,0579 & 0,1799 & 18,5120 & 0,0751 & 0,1618 & 16,9407 & 0,0309 \\
\hline Elman & 0,0834 & 8,8819 & 0,0296 & 0,1328 & 13,9725 & 0,0501 & 0,1765 & 18,3858 & 0,0687 & 0,1605 & 17,0611 & 0,0244 \\
\hline L-ANN & 0,0927 & 9,8636 & 0,0270 & 0,1406 & 14,9318 & 0,0408 & 0,1764 & 18,7244 & 0,0511 & 0,1643 & 17,9314 & 0,0054 \\
\hline RBF & 0,0884 & 9,3551 & 0,0365 & 0,1412 & 14,7057 & 0,0623 & 0,1858 & 19,1139 & 0,0848 & 0,1661 & 17,2954 & 0,0397 \\
\hline ANFIS & 0,0829 & 7,9212 & 0,0284 & 0,1192 & 11,0680 & 0,0410 & 0,1343 & 12,7252 & 0,0438 & 0,1215 & 11,9520 & 0,0114 \\
\hline ANFIS-2 & 0,0895 & 9,3282 & 0,0348 & 0,1367 & 14,4478 & 0,0567 & 0,1860 & 19,2294 & 0,0793 & 0,1672 & 17,5688 & 0,0336 \\
\hline
\end{tabular}

Table 5 Performance comparison of Çeşme wind speed time series for the test period 2

\begin{tabular}{|c|c|c|c|c|c|c|c|c|c|c|c|c|}
\hline & \multicolumn{9}{|c|}{1 month test } & \multicolumn{3}{|c|}{3 months test } \\
\hline & \multicolumn{3}{|c|}{6 hours } & \multicolumn{3}{|c|}{$12 \mathrm{~h}$} & \multicolumn{3}{|c|}{$24 \mathrm{~h}$} & \multicolumn{3}{|c|}{$24 h$} \\
\hline & IRMSE & NMAPE & Bias & NRMSE & NMAPE & Bias & NRMSE & NMAPE & Bias & NRMSE & NMAPE & Bias \\
\hline $\mathrm{P}^{(2)} \mathrm{AR}($ & 0436 & 3,5922 & 0,0033 & 591 & ,1160 & 0,0015 & 0,0789 & 7,5981 & $-0,0061$ & 0,0782 & 7,8179 & 0,0045 \\
\hline $\mathrm{AR}(3)$ & 11 & 5343 & 7 & 58 & 51 & 1 & 4 & 0624 & 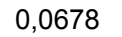 & 0972 & 0,2940 & 0,0773 \\
\hline MLFF & 0,0545 & 5,8218 & $-0,0058$ & 0,0785 & 8,3700 & $-0,0070$ & 0,1034 & 11,0578 & $-0,0094$ & 0,1059 & 11,3404 & 0,0102 \\
\hline MLP & 0580 & 6,2713 & $-0,0100$ & 0,0834 & 8,9522 & $-0,0133$ & 0,1086 & 11,6392 & $-0,0181$ & 0,1077 & 11,6112 & 0,0011 \\
\hline Elman & 5880 & 6,3409 & $-0,0117$ & 0,0842 & 9,1503 & $-0,0165$ & 1101 & 11,8817 & $-0,0$ & 0,1082 & 11,7624 & $-0,0013$ \\
\hline L-ANI & 5588 & 6,3336 & $-0,0089$ & 0,0848 & 9,1286 & $-0,0138$ & 0,1103 & 11,8562 & $-0,0186$ & 0,1086 & 11,7773 & 0,0007 \\
\hline RBF & 0,0580 & 6,6109 & $-0,0080$ & 0,0766 & 8,7526 & $-0,0040$ & 0,0963 & 10,6481 & 0,0032 & 0,1072 & 11,7528 & 0,0282 \\
\hline ANFIS & 0,0653 & 6,7592 & $-0,0040$ & 0,0927 & 9,7987 & $-0,0060$ & 0,1167 & 12,2866 & $-0,0068$ & 0,1171 & 12,5301 & 0,0141 \\
\hline ANFIS-2 & 0,0665 & 6,4903 & $-0,0092$ & 0,0889 & 9,0766 & $-0,0107$ & 0,1107 & 11,4970 & $-0,0093$ & 0,1097 & 11,6950 & 0,0125 \\
\hline
\end{tabular}

Table 6 Performance comparison of Bandon wind speed time series for the test period 2

\begin{tabular}{|c|c|c|c|c|c|c|c|c|c|c|c|c|}
\hline & \multicolumn{9}{|c|}{1 month test } & \multicolumn{3}{|c|}{3 months test } \\
\hline & & $6 \mathrm{~h}$ & & & $12 \mathrm{~h}$ & & & $24 \mathrm{~h}$ & & & $24 h$ & \\
\hline & NRMSE & NMAPE & Bias & NRMSE & NMAPE & Bias & NRMSE & NMAPE & Bias & NRMSE & NMAPE & Bias \\
\hline $\mathrm{P}^{(2)} \mathrm{AR}(2)$ & 0,0337 & 2,3707 & $-0,0004$ & 0,0496 & 3,9644 & $-0,0043$ & 0,0693 & 6,5320 & $-0,0148$ & 0,0878 & 7,1058 & 0,0015 \\
\hline $\operatorname{AR}(2)$ & 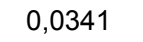 & 2 & ? & 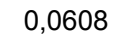 & ,7136 & - & & 77 & & 81 & 65 & 960 \\
\hline LFF & 728 & 9022 & $-0,0$ & 98 & 12,0676 & $-0,0$ & 55 & 5,7152 & -0 , & 41 & 29 & $-0,0213$ \\
\hline MLP & 0,0725 & 8,0474 & $-0,0288$ & ,1108 & 12,4097 & $-0,0433$ & 0,1393 & 16,1842 & $-0,0652$ & 0,1472 & 14,3800 & $-0,0214$ \\
\hline Elman & 0,0715 & 9189 & $-0,0270$ & 1080 & ,9932 & $-0,0368$ & ,1327 & 14,9218 & $-0,0$ & 0,1460 & 13,8345 & $-0,0020$ \\
\hline -ANN & 0,0853 & 10,2751 & $-0,0515$ & 0,1293 & 5,5128 & $-0,0769$ & 0,1568 & 18,7881 & $-0,0928$ & & 15,8369 & $-0,0475$ \\
\hline RBF & 0,0711 & 7,7178 & $-0,0253$ & 0,1073 & 11,6357 & $-0,0343$ & 0,1307 & 14,6585 & $-0,0471$ & 0,1439 & 13,5601 & $-0,0038$ \\
\hline ANFIS-1 & 0,0757 & 8,2571 & $-0,0306$ & 0,1105 & 12,2874 & $-0,0499$ & 0,1297 & 15,5006 & $-0,0782$ & 0,1311 & 13,2829 & $-0,0439$ \\
\hline ANFIS-2 & 0,0784 & 8,7240 & $-0,0315$ & 0,1196 & 13,6140 & $-0,0508$ & 0,1468 & 17,2387 & $-0,0738$ & 0,1527 & 15,1032 & $-0,0285$ \\
\hline
\end{tabular}



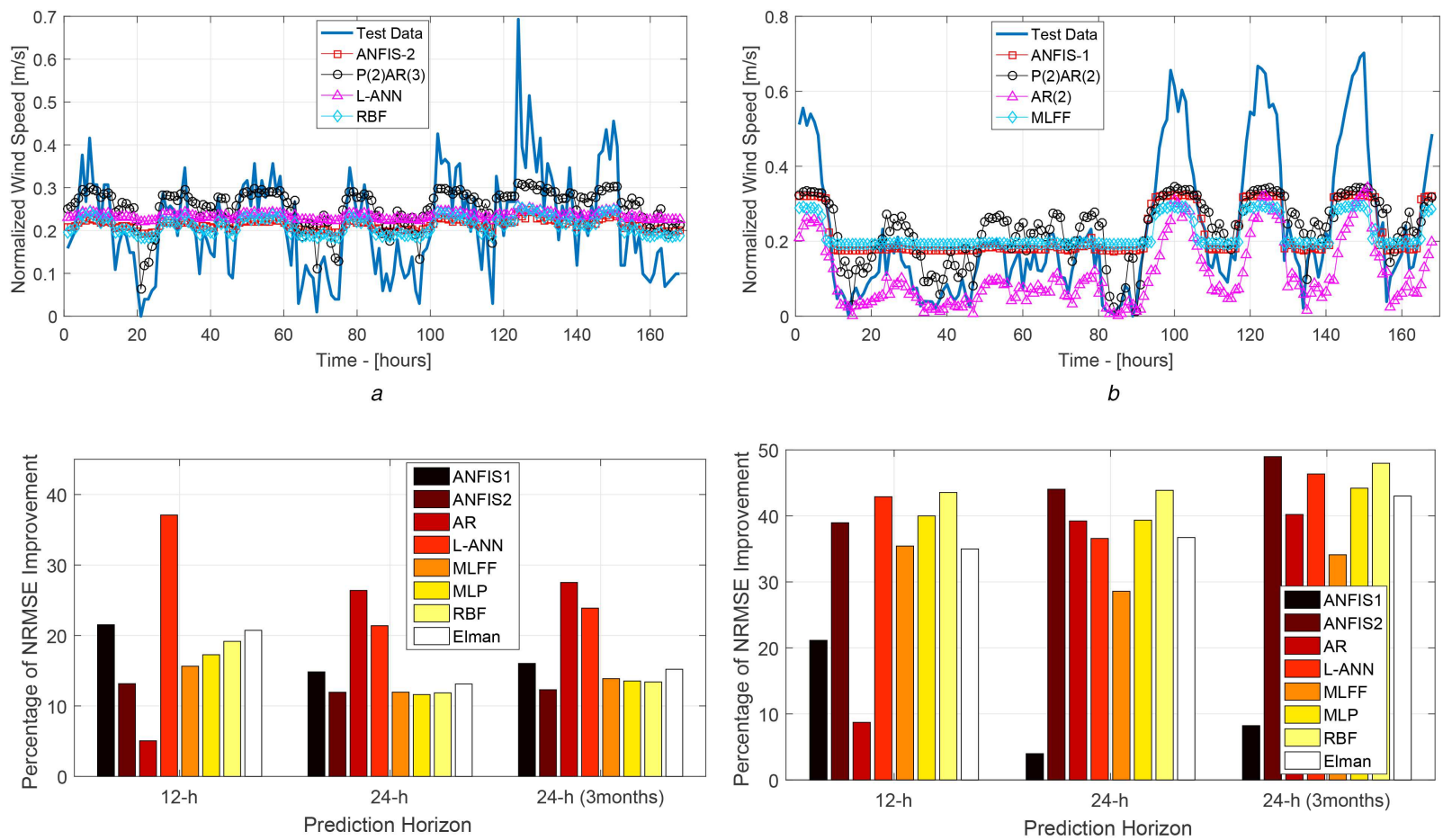

d

Fig. 4 Wind speed prediction comparison figures for the test period 1

(a) Çeşme (period 1) - $24 \mathrm{~h}$ ahead prediction, (b) Bandon (period 1) - $24 \mathrm{~h}$ ahead prediction, (c) Çeşme (period 1) - percentage of NRMSE improvement by PAR with respect to other models, (d) Bandon (period 1) - percentage of NRMSE improvement by PAR with respect to other models

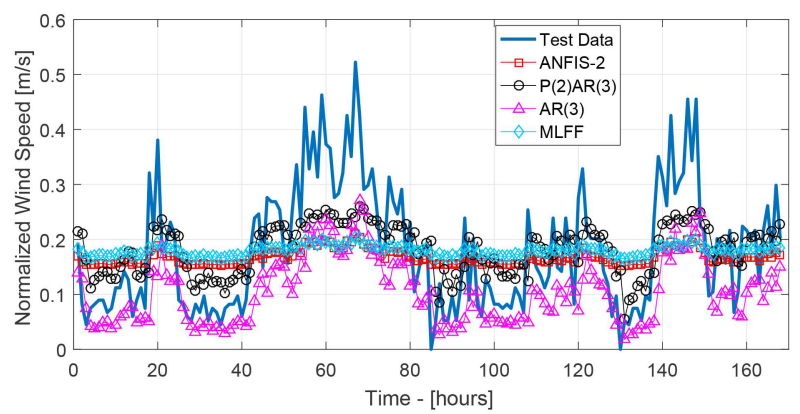

a

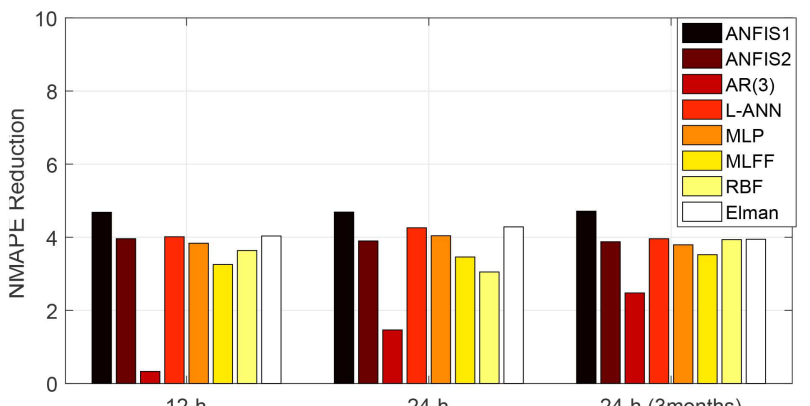

Prediction Horizon - [hours]

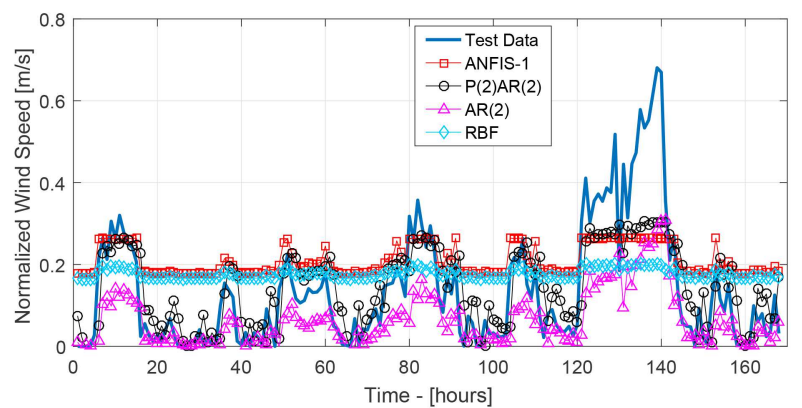

$b$

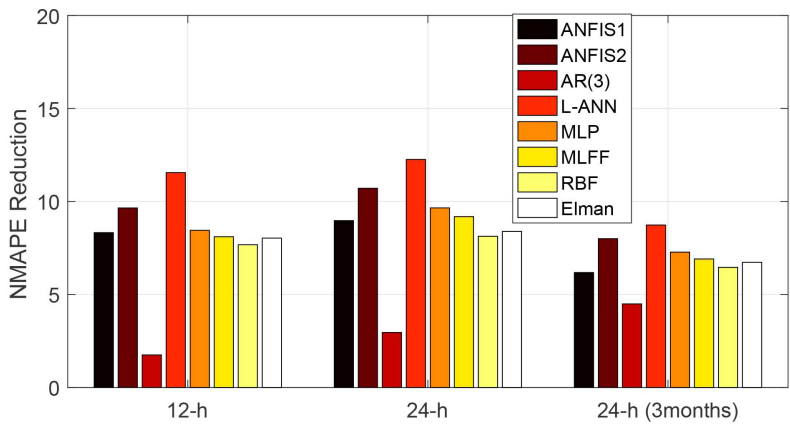

Prediction Horizon - [hours]

Fig. 5 Wind speed prediction comparison figures for the test period 2

(a) Çeşme (period 2) $-24 \mathrm{~h}$ ahead prediction, (b) Bandon (period 2) - $24 \mathrm{~h}$ ahead prediction, (c) Çeşme (period 2) - NMAPE reduction obtained using PAR with respect to other models, (d) Bandon (period 2) - NMAPE reduction obtained using PAR wrt other models

models. Observing these values, for all the prediction horizon values, PAR model improves the prediction performance in NMAPE by about $4 \%$ for Çeşme and $10 \%$ for Bandon.

\subsection{Wind power prediction results}

Examining Table 7, the performance of $\mathrm{P}^{(2)} \mathrm{AR}(1)$ in test period 1 is dominantly better than all other models for all the prediction horizons. Linear AR(1) and MLFF have achieved the second and third best performances, respectively, and they are preferable to all other models for a longer prediction horizon. For all the prediction horizons, most of the models have overestimated the wind power. Wind power prediction performances in test period 2 are shown in Table 8. As seen clearly, $\mathrm{P}^{(2)} \mathrm{AR}(1)$ performs better than all other models for all the prediction horizons generally. MLFF and MLP perform considerable for longer horizons. 
Table 7 Performance comparison of wind power prediction for test period 2

\begin{tabular}{|c|c|c|c|c|c|c|c|c|c|c|c|c|}
\hline & \multicolumn{9}{|c|}{1 month test } & \multicolumn{3}{|c|}{3 months test } \\
\hline & \multicolumn{3}{|c|}{$6 \mathrm{~h}$} & \multicolumn{3}{|c|}{$12 \mathrm{~h}$} & \multicolumn{3}{|c|}{$24 \mathrm{~h}$} & \multicolumn{3}{|c|}{$24 h$} \\
\hline & RMSE & NMAPE & Bias & NRMSE & NMAPE & Bias & NRMSE & NMAPE & Bias & NRMSE & NMAPE & Bias \\
\hline $\mathrm{P}^{(2)} \mathrm{AR}(1)$ & 0,0210 & 1,5283 & 0,0008 & 0,0359 & 2,7777 & $-0,0002$ & 0,0567 & 4,7827 & $-0,0040$ & 0,0913 & 5,5466 & 0,0129 \\
\hline $\mathrm{AR}(1)$ & 0311 & 2,8262 & 0,0215 & 0,0583 & 5,2799 & 0,0403 & ,1023 & 9,2787 & 0,0710 & 0,1284 & 9,7502 & 0,0904 \\
\hline MLFF & 462 & 2700 & 0,0275 & 0,0792 & 8,8320 & $-0,0512$ & 0,1124 & 12,2036 & $-0,0749$ & 0,1163 & 10,0737 & $-0,0520$ \\
\hline MLP & 4417 & 4,6074 & $-0,0207$ & 0,0713 & 8,2005 & $-0,0427$ & 0,1 & 12,6027 & $-0,0714$ & 0,1169 & 10,6537 & $-0,0504$ \\
\hline Elman & 9 & 14 & $-0,0$ & 88 & 10,2639 & $-0,0$ & & 17,0304 & $-0,0$ & 0,1 & 14,8224 & $-0,0477$ \\
\hline L-ANN & 461 & 1694 & $-0,0173$ & 0,0804 & 9,0109 & $-0,0301$ & 0,1249 & 14,0076 & $-0,0469$ & 0,1450 & 12,5989 & $-0,0159$ \\
\hline RBF & 5003 & 5,8857 & $-0,0241$ & 0,0943 & 11,0839 & $-0,0498$ & 0,1537 & 17,9750 & $-0,0874$ & 0,1693 & 15,5102 & $-0,0533$ \\
\hline ANFIS-1 & 0,0672 & 7,2553 & $-0,0228$ & 0,1131 & 12,3731 & $-0,0429$ & 0,1435 & 15,4991 & $-0,0575$ & 0,1723 & 14,1025 & $-0,0208$ \\
\hline ANFIS-2 & 0,0689 & 7,4776 & $-0,0214$ & 0,1155 & 12,6548 & $-0,0360$ & 0,1584 & 17,7685 & $-0,0599$ & 0,1870 & 16,0925 & $-0,0199$ \\
\hline
\end{tabular}

Table 8 Performance comparison of wind power prediction for test period 1

\begin{tabular}{|c|c|c|c|c|c|c|c|c|c|c|c|c|}
\hline & \multicolumn{9}{|c|}{ 1-month test } & \multicolumn{3}{|c|}{ 3-months test } \\
\hline & \multicolumn{3}{|c|}{$6 \mathrm{~h}$} & \multicolumn{3}{|c|}{$12 \mathrm{~h}$} & \multicolumn{3}{|c|}{$24 \mathrm{~h}$} & \multicolumn{3}{|c|}{$24 h$} \\
\hline & IRMSE & NMAPE & Bias & NRMSE & NMAPE & Bias & NRMSE & NMAPE & Bias & NRMSE & NMAPE & Bias \\
\hline $\mathrm{P}^{(2)} \mathrm{AR}(1)$ & 0,0519 & 2,6401 & 0,0165 & 0833 & 4,4254 & 0,0259 & 0,1199 & 6,8146 & 0,0345 & 0,1467 & 9,1705 & 0,0548 \\
\hline $\mathrm{AR}(1)$ & 1 & 2898 & 0,0306 & 43 & 1249 & 2 & 33 & 62 & 0,1006 & 726 & 3679 & 1263 \\
\hline MLFF & 0,0633 & 5,5255 & $-0,0132$ & 0,1019 & 8,8270 & $-0,0320$ & 0,1332 & 11,6392 & $-0,0535$ & 0,1316 & 11,0963 & $-0,0245$ \\
\hline MLP & 0596 & 5,2008 & $-0,0085$ & 0,1002 & 8,6963 & $-0,0203$ & 0,1336 & 11,8839 & $-0,0373$ & 0,1405 & 12,0112 & $-0,0078$ \\
\hline Elman & 662 & 5,7053 & $-0,0026$ & 0,1198 & 10,4319 & $-0,0099$ & 898 & 16,8847 & $-0,0289$ & 0,2070 & 17,8914 & 0,0136 \\
\hline L-ANN & 625 & 5,4174 & $-0,0006$ & 0,1095 & 9,4819 & $-0,0009$ & 0,1715 & 14,8508 & $-0,0014$ & 0,1896 & 15,8575 & 0,0389 \\
\hline RBF & 0,0687 & 5,9576 & $-0,0054$ & 0,1244 & 11,0212 & $-0,0170$ & 0,1916 & 17,2038 & $-0,0333$ & 0,2050 & 17,8344 & 0,0105 \\
\hline ANFIS-1 & 0,1084 & 9,2020 & 0,0064 & 0,1751 & 15,2000 & 0,0017 & 0,2408 & 20,8790 & $-0,0106$ & 0,2682 & 22,5564 & 0,0441 \\
\hline ANFIS-2 & 0,1094 & 9,2455 & 0,0112 & 0,1828 & 15,7807 & 0,0133 & 0,2456 & 21,0580 & 0,0088 & 0,2739 & 22,6919 & 0,0656 \\
\hline
\end{tabular}

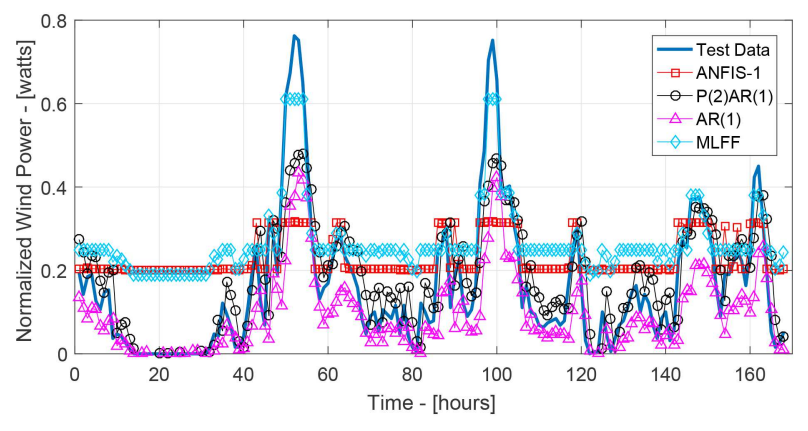

a

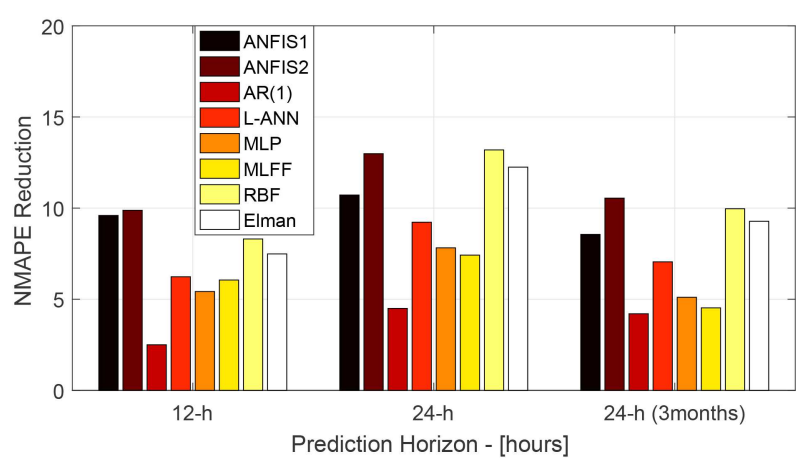

$c$

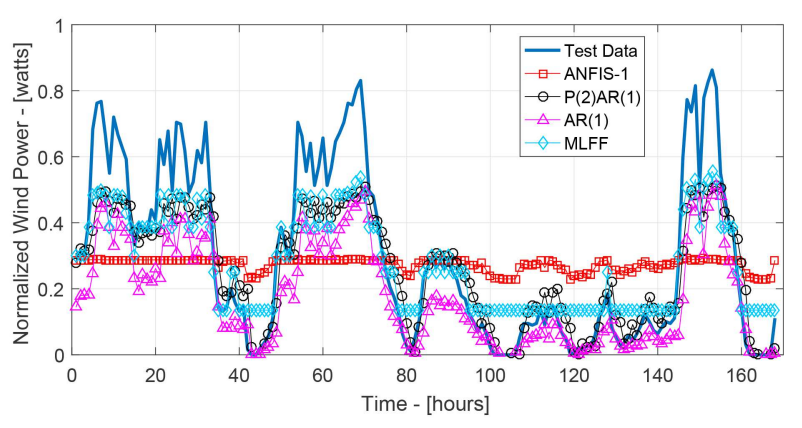

b

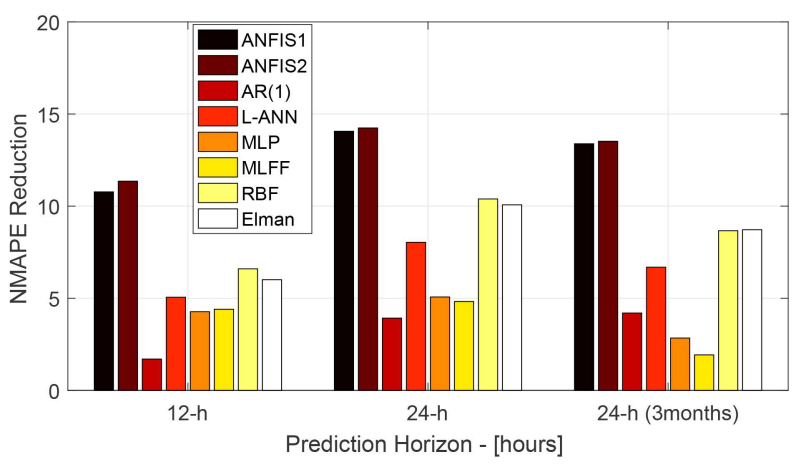

d

Fig. 6 Wind power prediction comparison figures

(a) Test period $1-24 \mathrm{~h}$ ahead prediction, (b) Test period $2-24 \mathrm{~h}$ ahead prediction, (c) Test period 1 - NMAPE reduction obtained using PAR with respect toother models, (d) Test period 2 - NMAPE reduction obtained using PAR with respect toother models

Figs. $6 a$ and $b$ show the $24 \mathrm{~h}$ ahead wind power prediction results versus observed test data in a week. Examining these figures, PAR and MLFF capture the test data trend better than any other model for $24 \mathrm{~h}$ of prediction horizon. PAR captures lowerpower values better than MLFF, when MLFF is better for higherpower values. Performance of ANFIS and linear models fall behind
PAR for both test periods. Figs. $6 c$ and $d$ show the NMAPE improvement of PAR over all other models. For $24 \mathrm{~h}$ ahead prediction, NMAPE values of PAR are around 5-10\%; however, the rest are around 10-20\%. Thus, improvement of PAR is at least $5 \%$ for both seasons. 
Examining the error metrics for 1 and 3 months, the results generally follow the same characteristics. Increasing the test length did not improve the performance of the proposed method and the others. However, using two different test length proves the applicability of the proposed method for whole year.

\section{Discussion and conclusion}

A detailed comparative study has been carried out for PAR model performance on wind speed/power predictions up to $24 \mathrm{~h}$ ahead of time over several ANN, ANFIS and linear AR models. Two data sets for two different locations in Turkey and USA are used to train and test the models. Results are presented in both figures and performance metrics such as NRMSE, NMAPE and bias to enrich the conclusion of this paper.

In particular, wind speed prediction results are similar to many other studies in the literature which claim that the prediction performance decreases when prediction horizon increases. In addition, linear model performance for longer prediction horizon gets worst than non-linear models. This fact reveals that non-linear characteristics of wind speed are more visible when prediction horizon is longer. Examining the performance metric values, linear $\mathrm{AR}$ achieves better wind speed prediction results in the first $6-10 \mathrm{~h}$ of prediction horizon, whereas PAR appears to be more successful for prediction horizons over 6-10 h. RBF, MLFF and MLP show close but inferior performance to PAR for longer horizons. Both ANFIS models achieve similar performance with most of the ANN. Performances of ANN and ANFIS are relatively worst when test period 2 is used for predictions and did not even reach the performance of linear AR.

The proposed method has been used also in wind power predictions. Examining the wind power prediction results shows us that performance of PAR has been distinctive and it outperforms other models for all the cases. Although MLFF and MLP perform worst than PAR, they appear better when compared with the other ANN and ANFIS models. Examining bias values of all the models for power prediction, we can conclude that over or under estimation of the wind power changes according to the data set in use. Specifically, PAR models generally underestimate the power in test period 1 and overestimate in test period 2. Moreover, for increasing order of prediction horizon values, bias values for PAR models generally follow a slightly increasing characteristic and for all the data sets bias values are between -0.05 and 0.05 . Apart from its performance with respect to NRMSE and NMAPE for all the data sets, PAR performs predictions with very low bias values. In particular, PAR has the lowest bias for $24 \mathrm{~h}$ ahead predictions in some of the cases, and in the other cases, it is among the best five methods in terms of bias values.

Moreover, PAR model is computationally more efficient than other non-linear models which are used in this paper. PAR models have very low number of adjustable parameters (close to the linear models) and, however, achieve the best results for a prediction horizon values higher than $6 \mathrm{~h}$. This superior performance makes PAR more preferable compared with the models which have widespread usage in wind speed/power prediction studies.

We should state that the non-linear functions that have been employed by ANN and ANFIS can be represented by polynomials with infinite order. However, PAR prediction performance shows that lower degree polynomials (order 2 for this paper) is enough for $24 \mathrm{~h}$ ahead wind speed/power predictions. Thus, we are of the opinion that the effects of these higher-ordered polynomial components in non-linear functions of ANN and ANFIS cause failure on prediction results.

\section{References}

[1] Maatallah, O., Achuthan, A., Janoyan, K., et al.: 'Recursive wind speed forecasting based on Hammerstein auto-regressive model', Appl. Energy, 2015, 145, pp. 191-197

[2] Soman, S.S., Zareipour, H., Malik, O., et al. 'A review of wind power and wind speed forecasting methods with different time horizons'. North American Power Symp. (NAPS) 2010, 2010, pp. 1-8

[3] Kariniotakis, G., Pinson, P., Siebert, N., et al.: 'The state of the art in shortterm prediction of wind power - from an offshore perspective'. Proc., 2004 , pp. 20-21
[4] Colak, I., Sagiroglu, S., Yesilbudak, M.: 'Data mining and wind power prediction: a literature review', Renew. Energy, 2012, 46, pp. 241-247

[5] Fang, S., Chiang, H.-D.: 'Improving supervised wind power forecasting models using extended numerical weather variables and unlabelled data', IET Renew. Power Gener., 2017, 10, (10), pp. 1616-1624

[6] Haque, A.U., Mandal, P., Meng, J., et al.: 'Wind speed forecast model for wind farm based on a hybrid machine learning algorithm', Int. J. Sustain. Energy, 2015, 34, (1), pp. 38-51

[7] Nielsen, T., Joensen, A., Madsen, H., et al.: 'A new reference for wind power forecasting', Wind Energy, 1998, 1, (1), pp. 29-34

[8] Palomares-Salas, J., De la Rosa, J., Ramiro, J., et al.: 'Comparison of models for wind speed forecasting'. Proc. Int. Conf. Computational Science, 2009

[9] Gomes, P., Castro, R.: 'Wind speed and wind power forecasting using statistical models: autoregressive moving average (ARMA) and artificial neural networks (ANN)', Int. J. Sustain. Energy Dev., 2012, 1, (1/2), pp. 275 293

[10] Torres, J., Garcia, A., De Blas, M., et al: 'Forecast of hourly average wind speed with ARMA models in Navarre (Spain)', Sol. Energy, 2005, 79, (1), pp. $65-77$

[11] Haque, A., Mandal, P., Kaye, M., et al.: 'A new strategy for predicting shortterm wind speed using soft computing models', Renew. Sustain. Energy Rev. 2012, 16, (7), pp. 4563-4573

[12] Doucoure, B., Agbossou, K., Cardenas, A.: 'Time series prediction using artificial wavelet neural network and multi-resolution analysis: application to wind speed data', Renew. Energy, 2016, 92, pp. 202-211

[13] De Giorgi, M., Ficarella, A., Tarantino, M.: 'Error analysis of short term wind power prediction models', Appl. Energy, 2011, 88, (4), pp. 1298-1311

[14] Renani, E.T., Elias, M.F.M., Rahim, N.A.: 'Using data-driven approach for wind power prediction: a comparative study', Energy Convers. Manage., 2016, 118, pp. 193-203

[15] Katsigiannis, Y., Tsikalakis, A., Georgilakis, P. et al.: 'Improved wind power forecasting using a combined neuro-fuzzy and artificial neural network model', Adv. Artif. Intell., 2006, 1, pp. 105-115, (Springer)

[16] Kusiak, A., Zheng, H., Song, Z.: 'Short-term prediction of wind farm power a data mining approach', IEEE Trans. Energy Convers., 2009, 24, (1), pp. $125-136$

[17] Catalao, J., Pousinho, H., Mendes, V.: 'Hybrid intelligent approach for shortterm wind power forecasting in Portugal', IET Renew. Power Gener., 2011, 5, (3), pp. 251-257

[18] Fang, S., Chiang, H.-D.: 'A high-accuracy wind power forecasting model', IEEE Trans. Power Syst., 2016, 32, (2), pp. 1589-1590

[19] Fugon, L., Juban, J., Kariniotakis, G.: 'Data mining for wind power forecasting'. European Wind Energy Conf. \& Exhibition EWEC 2008, 2008, p. 6

[20] Kusiak, A., Zhang, Z: 'Short-horizon prediction of wind power: a data-driven approach', IEEE Trans. Energy Convers., 2010, 25, (4), pp. 1112-1122

[21] Lee, D.: 'Short-term prediction of wind farm output using the recurrent quadratic Volterra model'. 2011 IEEE Power and Energy Society General Meeting, 2011, pp. 1-8

[22] Lydia, M., Kumar, S.S., Selvakumar, A.I. et al. 'Linear and non-linear autoregressive models for short-term wind speed forecasting', Energy Convers. Manage., 2016, 112, pp. 115-124

[23] Cadenas, E., Rivera, W., Campos-Amezcua, R., et al.: 'Wind speed prediction using a univariate arima model and a multivariate NARX model', Energies, 2016, 9, (2), p. 109

[24] Lahaye, P.-J., Poline, J.-B., Flandin, G., et al.: 'Functional connectivity: studying nonlinear, delayed interactions between BOLD signals', Neuroimage, 2003, 20, (2), pp. 962-974

[25] Gruber, J., Bordons, C., Bars, R., et al. 'Nonlinear predictive control of smooth nonlinear systems based on Volterra models. application to a pilot plant', Int. J. Robust Nonlinear Control, 2010, 20, (16), pp. 1817-1835

[26] Fernandes, C., Mota, J., Favier, G., et al.: 'MIMO Volterra modeling for nonlinear communication channels', Learn. Nonlinear Models,2010, 2, (8), pp. 71-92

[27] Bekleric, S.: 'Nonlinear prediction via Volterra series and applications to geophysical data', Masters Abstr. Int., 2008, 47

[28] Kuruoğlu, E.: 'Nonlinear least LP-norm filters for nonlinear autoregressive $\alpha$ stable processes', Digit. Signal Process., 2002, 12, (1), pp. 119-142

[29] Karakuş, O., Kuruoğlu, E., Altinkaya, M.: 'Long term wind speed prediction with polynomial autoregressive model'. 2015 23th Signal Processing and Communications Applications Conf. (SIU), 2015, pp. 645-648

[30] Hong, T., Pinson, P., Fan, S.: 'Global energy forecasting competition 2012', Int. J. Forecast., 2014, 30, (2), pp. 357-363

[31] Sfetsos, A.: 'A comparison of various forecasting techniques applied to mean hourly wind speed time series', Renew. Energy, 2000, 21, (1), pp. 23-35

[32] Takagi, T., Sugeno, M.: 'Fuzzy identification of systems and its applications to modeling and control', IEEE Trans. Syst. Man Cybern., 1985, SMC-15, (1), pp. 116-132

[33] Abraham, A.: 'Adaptation of fuzzy inference system using neural learning', in Nedjah, N., de Macedo Mourelle, L. (Eds.): 'Fuzzy systems engineering' (Springer, 2005), pp. 53-83

[34] Bernardo-Faura, M., Massen, S., Falk, C et al: 'Data-derived modeling characterizes plasticity of MAPK signaling in melanoma', PLoS Comput. Biol., 2014, 10, (9), pp. 1-18

[35] Cheng, H., Tan, P.-N., Gao, J., et al.: 'Multistep-ahead time series prediction', in Ng, W. -K., Kitsuregawa, M., Li, J., et al. (Eds.): 'Advances in knowledge discovery and data mining' (Springer, 2006), pp. 765-774

[36] Bard, Y.: 'Nonlinear parameter estimation' (Academic Press, 1974)

[37] Harper, B., Kepert, J., Ginger, J.: 'Guidelines for converting between various wind averaging periods in tropical cyclone conditions'. (World Meteorological Organization, WMO/TD), 2010, 1555, pp. 1-54 
[38] 'Agrimet-Cooperative Agricultural Weather Network'. Available at http:// www.usbr.gov/pn/agrimet/webaghrread.html, accessed 10 August 2016
[39] Karakus, O., Kuruoglu, E.E., Altinkaya, M.A.: 'Estimation of the nonlinearity degree for polynomial autoregressive processes with RJMCMC'. 23rd European Signal Processing Conf. (EUSIPCO), 2015, pp. 953-957 